\title{
On representations of spinor genera
}

\author{
Wai Kiu Chan and Fei Xu
}

\begin{abstract}
We determine exactly when a quadratic form is represented by a spinor genus of another quadratic form of three or four variables. We apply this to extend the embedding theorem for quaternion and also answer a question by Borovoi.
\end{abstract}

\section{Introduction}

It is a basic question to decide when a quadratic form is represented by the spinor genus of another quadratic form. Some effort has been made in [EH82] and [HSX98]. In this paper, we make some progress on this subject. As an application, we show that the result in [EH82] is a special case of our investigation. There are two other motivations for this paper. First of all, we explain that the embedding theorem for quaternion algebras proved in [CF99] is a consequence of the representation theory of ternary quadratic forms and hence, in principle, the main results in [CF99] can be extended to arbitrary orders. To illustrate our point, we generalize Theorem 3.3 in [CF99] to Eichler orders. The second motivation is to explain how the results in [Xu01] are related to those in [BR95], and answer a question raised in [Bor01] concerning the representation mass of an integer by indefinite ternary quadratic forms over $\mathbb{Z}$.

The notation and terminology are standard if not explained, or adopted from [Xu01], [Ome73] and [HSX98]. Let $V$ be a quadratic space over a number field $F$ with a non-degenerate symmetric bilinear form $B(x, y)$. The quadratic map on $V$ is denoted by $Q$ and its special orthogonal group by $S O(V)$. Let $\mathfrak{o}_{F}$ be the ring of integers of $F$. For any prime $\mathfrak{p}$ of $F, V_{\mathfrak{p}}$ (respectively $F_{\mathfrak{p}}$, etc.) denotes the local completion of $V$ (respectively $F$, etc.). If $\mathfrak{p}$ is a finite prime, the group of units of $\mathfrak{o}_{F_{\mathfrak{p}}}$ is denoted by $\mathfrak{u}_{\mathfrak{p}}$, and $\pi_{\mathfrak{p}}$ is a uniformizer of $F_{\mathfrak{p}}$. For any two elements $a, b \in F_{\mathfrak{p}}^{\times},(a, b)_{\mathfrak{p}}$ is the Hilbert symbol. Let $S O_{\mathrm{A}}(V)$ be the adèlic group of $S O(V), \theta_{\mathrm{A}}=\prod_{\mathfrak{p} \in \Omega} \theta_{\mathfrak{p}}$ be the adèlic spinor norm map of $S O_{\mathrm{A}}(V)$, and $\mathbf{I}_{F}$ (respectively $\mathbf{A}_{F}$ ) be the group of idèles (respectively adèles) of $F$. For an $\mathfrak{o}_{F}$-lattice $L$ on $V$, gen $(L)$ (respectively $\operatorname{spn}(L)$ and $\operatorname{cls}(L)$ ) is defined as the orbit of $L$ under the natural action of $S O_{\mathrm{A}}(V)$ (respectively $S O(V) \operatorname{ker} \theta_{\mathrm{A}}$ and $S O(V)$ ), and $S O_{\mathrm{A}}(L)$ is the stabilizer of $L$ under the action of $S O_{\mathrm{A}}(V)$. We also use $\mathfrak{n}(L), \mathfrak{s}(L)$ and $\mathfrak{v}(L)$ to denote scale, norm and volume of $L$, respectively. Throughout this paper, all scales of lattices are integral. For two $\mathfrak{o}_{F}$-modules $L_{1} \subseteq L_{2}$ of the same rank, $\left[L_{2}: L_{1}\right]$ is the module index ideal.

For any two lattices $K$ and $L$ in $\mathrm{V}$, define

$$
X_{\mathrm{A}}(L / K)=\left\{\sigma_{\mathrm{A}} \in S O_{\mathrm{A}}(V): K \subseteq \sigma_{\mathrm{A}} L\right\}
$$

and

$$
X\left(L_{\mathfrak{p}} / K_{\mathfrak{p}}\right)=\left\{\sigma \in S O\left(V_{\mathfrak{p}}\right): K_{\mathfrak{p}} \subseteq \sigma L_{\mathfrak{p}}\right\} .
$$

It is clear that $X_{\mathrm{A}}(L / K)$ (respectively $\left.X\left(L_{\mathfrak{p}} / K_{\mathfrak{p}}\right)\right)$ is non-empty if and only if $K$ (respectively $K_{\mathfrak{p}}$ ) is represented by gen $(L)$ (respectively $L_{\mathfrak{p}}$ ).

Received 23 November 2001, accepted in final form 26 June 2003.

2000 Mathematics Subject Classification 11Exx (primary); 11Fxx, 11Gxx (secondary).

Keywords: spinor genera, representation mass, Hardy-Littlewood varieties.

This journal is (C) Foundation Compositio Mathematica 2004. 


\section{W. K. Chan AND F. XU}

\section{Representation by spinor genera I, codimension less than or equal to one}

Suppose $K$ is represented by $\operatorname{gen}(L)$. Then there exists

$$
\sigma_{\mathrm{A}} \in S O_{\mathrm{A}}(V) \text { such that } K \subseteq \sigma_{\mathrm{A}} L .
$$

By the reduction formulae for computing the relative spinor norms in [HSX98] and [Xu99], $\theta_{\mathrm{A}}\left(X\left(\sigma_{\mathrm{A}} L / K\right)\right)$ depends only on the Jordan splittings of $K_{\mathfrak{p}}$ and $L_{\mathfrak{p}}$ for all $\mathfrak{p}<\infty$ and is independent of the choice of $\sigma_{\mathrm{A}}$.

Definition 2.1. $\theta_{\mathrm{A}}(\operatorname{gen}(L): K)$ is defined as $\theta_{\mathrm{A}}\left(X\left(\sigma_{\mathrm{A}} L / K\right)\right)$.

By [HSX98] or [Xu99], $K$ is represented by $\operatorname{spn}(L)$ if and only if

$$
\theta_{\mathrm{A}}\left(\sigma_{\mathrm{A}}\right) \in F^{\times} \theta_{\mathrm{A}}(\operatorname{gen}(L): K) .
$$

We extended some results in [HSX98] to the representation setting for low-dimensional cases.

Proposition 2.3. Suppose $K_{\mathfrak{p}}$ and $L_{\mathfrak{p}}$ are unimodular and $\left[K_{\mathfrak{p}}: K_{\mathfrak{p}} \cap L_{\mathfrak{p}}\right]=\mathfrak{p}^{r}$ for some non-dyadic prime p. Furthermore:

1) $\operatorname{rank}(L)=\operatorname{rank}(K)$; or

2) $\operatorname{rank}(K)+1=\operatorname{rank}(L) \leqslant 4$.

Then $\pi_{\mathfrak{p}} \in \theta_{\mathfrak{p}}\left(X\left(L_{\mathfrak{p}} / K_{\mathfrak{p}}\right)\right)$ if and only if $r$ is odd.

Proof. 1) The argument is already in [HSX98].

2) We separate the discussion according to the rank of $L$. We present the proofs for the quaternary and ternary cases in below, and leave that for the binary case to the readers.

2i) $\operatorname{rank}\left(L_{\mathfrak{p}}\right)=3$ and $\operatorname{rank}\left(K_{\mathfrak{p}}\right)=2$. Write $B(K, L) \mathfrak{o}_{F_{\mathfrak{p}}}=\mathfrak{p}^{-s}$. Then $s \geqslant 0$. When $s=0, K_{\mathfrak{p}}$ splits $L_{\mathfrak{p}}$ and the proposition is obvious. Therefore, we assume that $s>0$.

Let $K=\mathfrak{o}_{F_{\mathfrak{p}}} x \perp \mathfrak{o}_{F_{\mathfrak{p}}} y$ and $B(x, L) \mathfrak{o}_{F_{\mathfrak{p}}}=\mathfrak{p}^{-s}$. Write

$$
L_{\mathfrak{p}}=\left(\mathfrak{o}_{F_{\mathfrak{p}}} e+\mathfrak{o}_{F_{\mathfrak{p}}} f\right) \perp \mathfrak{o}_{F_{\mathfrak{p}}} g, \quad Q(e)=Q(f)=0, \quad B(e, f)=1 \quad \text { and } \quad Q(g) \in \mathfrak{u}_{\mathfrak{p}} .
$$

Let $a, b$, and $c$ be in $F_{\mathfrak{p}}$ so that $x=a e+b f+c g$. Then $\min \{\operatorname{ord}(a), \operatorname{ord}(b), \operatorname{ord}(c)\}=-s$. If ord $(c)<0$, then $\operatorname{ord}(a)+\operatorname{ord}(b)=2 \operatorname{ord}(c)$. This implies that

$$
\min \{\operatorname{ord}(a), \operatorname{ord}(b)\}=\min \{\operatorname{ord}(a), \operatorname{ord}(b), \operatorname{ord}(c)\}=-s .
$$

Without loss of generality, we can assume that $\operatorname{ord}(a)=-s$. Then $x=\pi_{\mathfrak{p}}^{-s}(\alpha e+\beta f+\gamma g)$, where $\alpha \in \mathfrak{u}_{\mathfrak{p}}$ and $\beta$ and $\gamma$ are in $\mathfrak{o}_{F_{\mathfrak{p}}}$. Therefore, $\mathfrak{o}_{F_{\mathfrak{p}}} \pi_{\mathfrak{p}}^{s} x+\mathfrak{o}_{F_{\mathfrak{p}}} f$ splits $L_{\mathfrak{p}}$ and

$$
L_{\mathfrak{p}}=\left(\mathfrak{o}_{F_{\mathfrak{p}}} \pi_{\mathfrak{p}}^{s} x+\mathfrak{o}_{F_{\mathfrak{p}}} f\right) \perp \mathfrak{o}_{F_{\mathfrak{p}}} h \quad \text { for some } h \in L_{\mathfrak{p}} .
$$

If $y=\xi x+\eta f+\delta h$, then $\xi Q(x)+\eta \alpha \pi^{-s}=0$ and $y=\xi\left(x-\alpha^{-1} \pi^{s} Q(x) f\right)+\delta h$. Since

$$
\alpha \pi_{\mathfrak{p}}^{-s} \xi=\xi B(x, f)=B(y, f) \in \mathfrak{p}^{-s} \quad \text { and } \quad \delta^{2} Q(h)-\xi^{2} Q(x)=Q(y) \in \mathfrak{u}_{\mathfrak{p}},
$$

both $\xi$ and $\delta$ are in $\mathfrak{o}_{F_{\mathfrak{p}}}$, and at least one of $\xi$ and $\delta$ is a unit. As a result,

$$
L_{\mathfrak{p}} \cap K_{\mathfrak{p}}=\mathfrak{p}^{s} x+\mathfrak{o}_{F_{\mathfrak{p}}}(y-\xi x),
$$

and hence $r=s$.

Define $\sigma \in S O\left(V_{\mathfrak{p}}\right)$ by

$$
\sigma: \pi_{\mathfrak{p}}^{r}\left(x-\frac{1}{2} \alpha^{-1} Q(x) \pi_{\mathfrak{p}}^{r} f\right) \rightarrow x-\frac{1}{2} \alpha^{-1} Q(x) \pi_{\mathfrak{p}}^{r} f, f \rightarrow \pi_{\mathfrak{p}}^{r} f, h \rightarrow h .
$$

It is clear that

$$
\begin{aligned}
\sigma\left(L_{\mathfrak{p}}\right) & =\left(\mathfrak{o}_{F_{\mathfrak{p}}} \pi_{\mathfrak{p}}^{r} f+\mathfrak{o}_{F_{\mathfrak{p}}}\left(x-\frac{1}{2} \alpha^{-1} Q(x) \pi_{\mathfrak{p}}^{r} f\right)\right) \perp \mathfrak{o}_{F_{\mathfrak{p}}} h \\
& =\left(\mathfrak{o}_{F_{\mathfrak{p}}} x+\mathfrak{p}^{r} f\right) \perp \mathfrak{o}_{F_{\mathfrak{p}}} h \supset K_{\mathfrak{p}} .
\end{aligned}
$$




\section{REPRESENTATIONS OF SPINOR GENERA}

and $X\left(L_{\mathfrak{p}} / K_{\mathfrak{p}}\right)=\sigma S O\left(L_{\mathfrak{p}}\right)$. Since $\theta_{\mathfrak{p}}(\sigma) \in \pi_{\mathfrak{p}}^{r} \mathfrak{u}_{\mathfrak{p}}\left(F_{\mathfrak{p}}^{\times}\right)^{2}$, we conclude that $\pi_{\mathfrak{p}} \in \theta_{\mathfrak{p}}\left(X\left(L_{\mathfrak{p}} / K_{\mathfrak{p}}\right)\right)$ if and only if $r$ is odd.

2ii) $\operatorname{rank}\left(L_{\mathfrak{p}}\right)=4$ and $\operatorname{rank}\left(K_{\mathfrak{p}}\right)=3$. Without loss of generality, we can assume that $K_{\mathfrak{p}}=$ $\left(\mathfrak{o}_{F_{\mathfrak{p}}} x+\mathfrak{o}_{F_{\mathfrak{p}}} y\right) \perp \mathfrak{o}_{F_{\mathfrak{p}}} z$, where $Q(x)=Q(y)=0, B(x, y)=1$ and $B\left(K_{\mathfrak{p}}, L_{\mathfrak{p}}\right) \mathfrak{o}_{F_{\mathfrak{p}}}=B\left(x, L_{\mathfrak{p}}\right) \mathfrak{o}_{F_{\mathfrak{p}}}=\mathfrak{p}^{-s}$ with $s>0$. It is clear that $Q(z) \in \mathfrak{u}_{\mathfrak{p}}$. The isotropic vector $\mathfrak{p}^{s} x$ is primitive in $L_{\mathfrak{p}}$. Therefore, there are $f, u, v \in L_{\mathfrak{p}}$ such that

$$
L_{\mathfrak{p}}=\left(\mathfrak{p}^{s} x+\mathfrak{o}_{F_{\mathfrak{p}}} f\right) \perp\left(\mathfrak{o}_{F_{\mathfrak{p}}} u+\mathfrak{o}_{F_{\mathfrak{p}}} v\right) \quad \text { with } Q(f)=0 \text { and } B(x, f)=\pi_{\mathfrak{p}}^{-s} .
$$

If $y=a x+b f+c u+d v$, then

$$
a \pi_{\mathfrak{p}}^{-s}=a B(x, f)=B(y, f) \in \mathfrak{p}^{-s} \quad \text { and } \quad 1=B(x, y)=b B(x, f)=b \pi_{\mathfrak{p}}^{-s} .
$$

These imply that $a \in \mathfrak{o}_{F_{\mathfrak{p}}}$ and $b=\pi_{\mathfrak{p}}^{s}$. Similarly, we have $z=\alpha x+\beta u+\gamma v$ with $\alpha \in \mathfrak{o}_{F_{\mathfrak{p}}}$.

When $F_{\mathfrak{p}} u+F_{\mathfrak{p}} v$ is anisotropic, then

$$
\mathfrak{o}_{F_{\mathfrak{p}}} u+\mathfrak{o}_{F_{\mathfrak{p}}} v=\left\{x \in F_{\mathfrak{p}} u+F_{\mathfrak{p}} v: Q(x) \in \mathfrak{o}_{F_{\mathfrak{p}}}\right\} .
$$

Since

$$
Q(c u+d v)=-Q\left(a x+\pi_{\mathfrak{p}}^{s} f\right) \in \mathfrak{o}_{F_{\mathfrak{p}}} \quad \text { and } \quad Q(\beta u+\gamma v)=Q(z) \in \mathfrak{u}_{\mathfrak{p}},
$$

therefore $c, d, \beta$, and $\gamma$ are all in $\mathfrak{o}_{F_{\mathfrak{p}}}$ and

$$
K_{\mathfrak{p}} \cap L_{\mathfrak{p}}=\left(\mathfrak{p}^{s} x+\mathfrak{o}_{F_{\mathfrak{p}}}\left(\pi_{\mathfrak{p}}^{s} f+c u+d v\right)\right) \perp \mathfrak{o}_{F_{\mathfrak{p}}} z .
$$

It is clear that $\left[K_{\mathfrak{p}}: K_{\mathfrak{p}} \cap L_{\mathfrak{p}}\right]=\mathfrak{p}^{s}$. Define $\sigma \in S O\left(V_{\mathfrak{p}}\right)$ by $\sigma: x \rightarrow \pi_{\mathfrak{p}}^{-s} x, f \rightarrow \pi_{\mathfrak{p}}^{s} f, u \rightarrow u, v \rightarrow v$. Then $\sigma \in X\left(L_{\mathfrak{p}} / K_{\mathfrak{p}}\right)=\sigma S O\left(L_{\mathfrak{p}}\right)$ and we are done since $\theta_{\mathfrak{p}}(\sigma) \in \pi_{\mathfrak{p}}^{s} \mathfrak{u}_{\mathfrak{p}}\left(F_{\mathfrak{p}}^{\times}\right)^{2}$. Then

When $\mathfrak{o}_{F_{\mathfrak{p}}} u+\mathfrak{o}_{F_{\mathfrak{p}}} v$ is a hyperbolic plane, we can assume that $Q(u)=Q(v)=0$ and $B(u, v)=1$.

$$
c d=-a \in \mathfrak{o}_{F_{\mathfrak{p}}}, \quad \beta \gamma=2^{-1} Q(z) \in \mathfrak{u}_{\mathfrak{p}}, \quad \beta d+\gamma c=-\alpha \in \mathfrak{o}_{F_{\mathfrak{p}}} .
$$

Without loss of generality, we assume that $\operatorname{ord}(\beta) \leqslant 0$, and hence $\operatorname{ord}(\gamma) \geqslant 0$ and $\operatorname{ord}(d) \geqslant 0$.

If $\operatorname{ord}(c) \leqslant \operatorname{ord}(\beta)$, there is $\xi \in \mathfrak{o}_{F_{\mathfrak{p}}}$ such that $\xi c+\beta=0$. Then

$$
K_{\mathfrak{p}}=\mathfrak{o}_{F_{\mathfrak{p}}} x+\mathfrak{o}_{F_{\mathfrak{p}}}\left(\pi_{\mathfrak{p}}^{s} f+c u+d v\right)+\mathfrak{o}_{F_{\mathfrak{p}}}\left(\pi_{\mathfrak{p}}^{s} \xi f+(d \xi+\gamma) v\right)
$$

and

$$
K_{\mathfrak{p}} \cap L_{\mathfrak{p}}=\mathfrak{p}^{s} x+\mathfrak{p}^{-\operatorname{ord}(c)}\left(\pi_{\mathfrak{p}}^{s} f+c u+d v\right)+\mathfrak{o}_{F_{\mathfrak{p}}}\left(\xi \pi_{\mathfrak{p}}^{s} f+(\xi d+\gamma) v\right) .
$$

Therefore, $\left[K_{\mathfrak{p}}: K_{\mathfrak{p}} \cap L_{\mathfrak{p}}\right]=\mathfrak{p}^{s-\operatorname{ord}(c)}$. Let $\sigma \in S O\left(V_{\mathfrak{p}}\right)$ be defined by

$$
\sigma: x \rightarrow \pi_{\mathfrak{p}}^{-s} x, f \rightarrow \pi_{\mathfrak{p}}^{s} f, u \rightarrow \pi_{\mathfrak{p}}^{\text {ord }(c)} u, v \rightarrow \pi_{\mathfrak{p}}^{-\operatorname{ord}(c)} v
$$

Since

$$
d \xi+\gamma=2 d \xi+(\gamma-d \xi)=-2 a \xi c^{-1}-\alpha c^{-1} \in \mathfrak{p}^{-\operatorname{ord}(c)},
$$

we see that $K_{\mathfrak{p}} \subset L_{\mathfrak{p}}$ and $X\left(L_{\mathfrak{p}} / K_{\mathfrak{p}}\right)=\sigma S O\left(L_{\mathfrak{p}}\right)$. The lemma now follows because $\theta(\sigma) \in \pi_{\mathfrak{p}}^{s-\operatorname{ord}(c)} \mathfrak{u}_{\mathfrak{p}}$ $\left(F_{\mathfrak{p}}^{\times}\right)^{2}$.

If $\operatorname{ord}(\beta)<\operatorname{ord}(c)$, there is $\eta \in \mathfrak{o}_{F_{\mathfrak{p}}}$ such that $c+\eta \beta=0$. Then

$$
K_{\mathfrak{p}}=\mathfrak{o}_{F_{\mathfrak{p}}} x+\mathfrak{o}_{F_{\mathfrak{p}}}\left(\pi_{\mathfrak{p}}^{s} f+(d+\eta \gamma) v\right)+\mathfrak{o}_{F_{\mathfrak{p}}}(\beta u+\gamma v)
$$

and

$$
K_{\mathfrak{p}} \cap L_{\mathfrak{p}}=\mathfrak{p}^{s} x+\mathfrak{o}_{F_{\mathfrak{p}}}\left(\pi_{\mathfrak{p}}^{s} f+(d+\gamma \eta) v\right)+\mathfrak{p}^{-\operatorname{ord}(\beta)}(\beta u+\gamma v) .
$$

Therefore, $\left[K_{\mathfrak{p}}: K_{\mathfrak{p}} \cap L_{\mathfrak{p}}\right]=\mathfrak{p}^{s-\operatorname{ord}(\beta)}$. Define

$$
\sigma: x \rightarrow \pi_{\mathfrak{p}}^{-s} x, f \rightarrow \pi_{\mathfrak{p}}^{s} f, u \rightarrow \pi_{\mathfrak{p}}^{\operatorname{ord}(\beta)} u, v \rightarrow \pi_{\mathfrak{p}}^{-\operatorname{ord}(\beta)}
$$




\section{W. K. Chan AND F. XU}

Then

$$
\sigma \in X\left(L_{\mathfrak{p}} / K_{\mathfrak{p}}\right)=\sigma S O\left(L_{\mathfrak{p}}\right) \quad \text { and } \quad \theta_{\mathfrak{p}}(\sigma) \in \pi_{\mathfrak{p}}^{s-\operatorname{ord}(\beta)} \mathfrak{u}_{\mathfrak{p}}\left(F_{\mathfrak{p}}^{\times}\right)^{2} .
$$

Remark 2.4. Suppose that $K_{\mathfrak{p}}$ and $L_{\mathfrak{p}}$ are unimodular over some non-dyadic prime $\mathfrak{p}$. If $\operatorname{rank}\left(K_{\mathfrak{p}}\right) \leqslant$ $\operatorname{rank}\left(L_{\mathfrak{p}}\right) \leqslant 3$, then

$$
B\left(K_{\mathfrak{p}}, L_{\mathfrak{p}}\right) \mathfrak{o}_{F_{\mathfrak{p}}}=\mathfrak{p}^{-s} \text { if and only if }\left[K_{\mathfrak{p}}: K_{\mathfrak{p}} \cap L_{\mathfrak{p}}\right]=\mathfrak{p}^{s} .
$$

This statement is proved in the above proposition for the case in which $\operatorname{rank}\left(L_{\mathfrak{p}}\right)=3$ and $\operatorname{rank}\left(K_{\mathfrak{p}}\right)=2$. The remaining cases can be easily verified. It is clear that such a statement is not true when $\operatorname{rank}\left(L_{\mathfrak{p}}\right)>3$.

We would expect that Proposition 2.3 is true for all codimension one cases. A possible approach is to have a result like [Ome73, Theorem 91:2] for the codimension one situation. However, such a result is not true in general. We explain this point by the following example.

Example 2.5. Let $\mathfrak{p}$ be a non-dyadic prime and $L$ be the $\mathfrak{o}_{F_{\mathfrak{p}}}$-lattice

$$
\left(\mathfrak{o}_{F_{\mathfrak{p}}} e_{1}+\mathfrak{o}_{F_{\mathfrak{p}}} f_{1}\right) \perp\left(\mathfrak{o}_{F_{\mathfrak{p}}} e_{2}+\mathfrak{o}_{F_{\mathfrak{p}}} f_{2}\right),
$$

where $Q\left(e_{1}\right)=Q\left(e_{2}\right)=Q\left(f_{1}\right)=Q\left(f_{2}\right)=0$ and $B\left(e_{1}, f_{1}\right)=B\left(e_{2}, f_{2}\right)=1$. Let

$$
K_{\mathfrak{p}}=\left(\mathfrak{o}_{F_{\mathfrak{p}}} \pi^{r} e_{1}+\mathfrak{o}_{F_{\mathfrak{p}}} \pi^{-r}\left(f_{1}+\pi^{-2} e_{1}+\pi^{-1}\left(e_{2}-f_{2}\right)\right)\right) \perp \mathfrak{o}_{F_{\mathfrak{p}}}\left(e_{2}+f_{2}\right),
$$

where $r$ is a positive integer. We claim that there is no regular two-dimensional subspace $U$ such that $L_{\mathfrak{p}}=\left(L_{\mathfrak{p}} \cap U\right) \perp\left(L_{\mathfrak{p}} \cap U^{\perp}\right)$ and $K_{\mathfrak{p}}=\left(K_{\mathfrak{p}} \cap U\right) \perp\left(K_{\mathfrak{p}} \cap U^{\perp}\right)$. Assume the contrary that such a binary space $U$ exists. Then $U$ is either anisotropic or isotropic.

If $U$ is anisotropic, so is $U^{\perp}$. The maximal $\mathfrak{o}_{F_{\mathfrak{p}}}$-lattices on $U$ and $U^{\perp}$ are unique. So $L_{\mathfrak{p}} \cap U=$ $K_{\mathfrak{p}} \cap U$ and $L_{\mathfrak{p}} \cap U^{\perp} \supset K_{\mathfrak{p}} \cap U^{\perp}$. Then $K_{\mathfrak{p}} \subset L_{\mathfrak{p}}$, which is a contradiction.

If $U$ is isotropic, there are $x$ and $y$ in $U$ with $Q(x)=Q(y)=0$ and $B(x, y)=1$ such that $L_{\mathfrak{p}} \cap U=\mathfrak{p}^{-s} x+\mathfrak{p}^{s} y$ and $K_{\mathfrak{p}} \cap U=\mathfrak{o}_{F_{\mathfrak{p}}} x+\mathfrak{o}_{F_{\mathfrak{p}}} y$, where $s$ is an integer with $s \geqslant 0$. Since

$$
K_{\mathfrak{p}} \cap L_{\mathfrak{p}}=\left(\mathfrak{o}_{F_{\mathfrak{p}}} \pi_{\mathfrak{p}}^{r} e_{1}+\mathfrak{o}_{F_{\mathfrak{p}}}\left(\pi_{\mathfrak{p}}^{2} f_{1}+e_{1}+\pi_{\mathfrak{p}}\left(e_{2}-f_{2}\right)\right)\right) \perp \mathfrak{o}_{F_{\mathfrak{p}}}\left(e_{2}+f_{2}\right),
$$

we have $s=r+2$.

Let $v=\pi_{\mathfrak{p}}^{-r-2} x \in L_{\mathfrak{p}}$. Then $\pi_{\mathfrak{p}}^{r+2} v=x$ is a primitive vector in $K_{\mathfrak{p}} \cap L_{\mathfrak{p}}$. Write

$$
\pi_{\mathfrak{p}}^{r+2} v=a \pi_{\mathfrak{p}}^{r} e_{1}+b\left(\pi_{\mathfrak{p}}^{2} f_{1}+e_{1}+\pi_{\mathfrak{p}}\left(e_{2}-f_{2}\right)\right)+c\left(e_{2}+f_{2}\right)
$$

where $a, b$ and $c$ are in $\mathfrak{o}_{F_{\mathfrak{p}}}$, and at least one of them is a unit. We only need to consider the case in which $a$ is a unit. By comparing the coefficients of $e_{1}, e_{2}$ and $f_{2}$, we conclude

$$
\operatorname{ord}(b)=r, \quad \operatorname{ord}(c)=r+1, \quad \operatorname{ord}(\pi b+c) \geqslant r+2 \quad \text { and } \quad \operatorname{ord}(\pi b-c) \geqslant r+2 .
$$

This is impossible because $\mathfrak{p}$ is non-dyadic.

It is also clear that $L_{\mathfrak{p}} \cap\left(F_{\mathfrak{p}} K_{\mathfrak{p}}\right)$ is not unimodular by the above argument.

Now we come to the global situation. Let $S_{0}$ be the set of prime divisors of $2 \mathfrak{v}(K) \mathfrak{v}(L)$. By the weak approximation property for $S O(V)$, there is $M \in \operatorname{cls}(L)$ such that

$$
K_{\mathfrak{p}} \subseteq M_{\mathfrak{p}} \text { for all } \mathfrak{p} \in S_{0} .
$$

Let $r_{\mathfrak{p}}$ be an integer such that $\left[K_{\mathfrak{p}}: K_{\mathfrak{p}} \cap M_{\mathfrak{p}}\right]=\mathfrak{p}^{r_{\mathfrak{p}}}$. It is clear that $r_{\mathfrak{p}}=0$ for almost all $\mathfrak{p}$. Let $i_{\mathfrak{p}}$ be the idèle of which the $\mathfrak{p}$ component is $\pi_{\mathfrak{p}}^{r_{\mathfrak{p}}}$ and the others are 1 . Put $i(M, K)=\prod_{\mathfrak{p}<\infty} i_{\mathfrak{p}} \in \mathbf{I}_{F}$. The ideal associated to $i(M, K)$ is $\prod_{\mathfrak{p}<\infty} \mathfrak{p}^{r_{\mathfrak{p}}}$ which is a generalization of the intersection ideal defined in [HSX98]. 


\section{REPRESENTATIONS OF SPINOR GENERA}

Definition 2.7. Define

$$
\Im(\operatorname{cls}(L), K)=\{i(M, K): M \in \operatorname{cls}(L) \text { with }(2.6)\}
$$

The following result generalizes [HSX98, Theorem 3.1].

Theorem 2.8. Suppose $K$ is represented by $\operatorname{gen}(L)$ with $\operatorname{rank}(L) \geqslant 3$. Let $i \in \mathfrak{I}(\operatorname{cls}(L), K)$ and:

1) $\operatorname{rank}(\mathrm{K})=\operatorname{rank}(\mathrm{L})$; or

2) $\operatorname{rank}(\mathrm{K})+1=\operatorname{rank}(\mathrm{L}) \leqslant 4$.

Then $K$ is represented by $\operatorname{spn}(L)$ if and only if $i \in F^{\times} \theta_{\mathrm{A}}(\operatorname{gen}(L): K)$.

The proof follows from (2.2) and Proposition 2.3.

The method we develop here can be used to check if one form can be represented by the spinor genus of another form directly, without the presence of a third form with a certain property in the genus (see [HSX98]). We explain this by the following example taken from [Hsi99].

Example 2.9. Consider the binary form $\eta(x, y)=5 x^{2}+16 y^{2}$ and the ternary form $g(x, y, z)=$ $4 x^{2}+45 y^{2}+45 z^{2}-10 y z$. By [Ome58], it is clear that $\eta$ is represented by $\operatorname{gen}(g)$. We want to determine whether $\eta$ is represented by $\operatorname{spn}(g)$.

It is clear that $S_{0}=\{2,5\}$ in this case. By [HSX98] and [HSX],

$$
p \text {-component of } \theta_{\mathrm{A}}(\operatorname{gen}(g), \eta)= \begin{cases}\mathbb{Z}_{p}^{\times} \mathbb{Q}_{p}^{\times 2}, & \text { for } p \neq 2,5, \\ \mathbb{Q}_{5}^{\times 2} \cup 5 \mathbb{Q}_{5}^{\times 2}, & \text { for } p=5, \\ \mathbb{Q}_{2}^{\times 2} \cup 2 \mathbb{Q}_{2}^{\times 2} \cup 5 \mathbb{Q}_{2}^{\times 2} \cup 10 \mathbb{Q}_{2}^{\times 2}, & \text { for } p=2, \\ \mathbb{R}^{>0}, & \text { for } p=\infty .\end{cases}
$$

It can easily be checked that $\left[\mathbf{I}_{\mathbb{Q}}: \mathbb{Q}^{\times} \theta_{\mathrm{A}}(\operatorname{gen}(g), \eta)\right]=2$. By class field theory, the extension associated to $\mathbb{Q}^{\times} \theta_{\mathrm{A}}(\operatorname{gen}(g), \eta)$ is $\mathbb{Q}(\sqrt{-1})$.

Let $L=\mathbb{Z}[u, v, w]$ correspond to the form $4 x^{2}+45 y^{2}+45 z^{2}-10 y z$. Then $K=\mathbb{Z}[2 u, v / 3]$ corresponds to $\eta$ and $K_{p} \subseteq L_{p}$ for $p=2,5$ and $[K: L \cap K]=3 \mathbb{Z}$. Since 3 is inert in $\mathbb{Q}(\sqrt{-1}), \eta$ is not represented by $\operatorname{spn}(g)$ by Theorem 2.8 .

The genus of $g$ has four spinor genera (see [BH82]). For example, $h_{1}=4 x^{2}+25 y^{2}+80 z^{2}$ is in $\operatorname{gen}(g)$. To determine if $\operatorname{spn}\left(h_{1}\right)$ represents $\eta$, we can set $L=\mathbb{Z}[u, v, w]$, which corresponds to $h_{1}$ and $K=\mathbb{Z}[(5 u+3 v+w) / 9,(-4 u+w) / 3]$, which corresponds to $\eta$. It is clear that $K_{p} \subseteq L_{p}$ for $p=2,5$ and $[K: L \cap K]=9 \mathbb{Z}$. Since 9 is trivial in $\mathbb{Q}(\sqrt{-1})$ under the Artin map, $\eta$ is represented by $\operatorname{spn}\left(h_{1}\right)$ by Theorem 2.8. As was pointed out in [Hsi99], $5 x^{2}+16 y^{2}+100 z^{2}$, a form in $\operatorname{spn}\left(h_{1}\right)$ by Kneser's neighborhood method at $p=13$, obviously represents $\eta$.

There are two more spinor genera in gen $(g)$. One contains $4 x^{2}+5 y^{2}+400 z^{2}$ and hence it obviously represents $\eta$. The other contains $16 x^{2}+20 y^{2}+25 z^{2}$ and it should not represent $\eta$, since the number of spinor genera in gen $(g)$ representing a given form is a power of two, see [HSX98]. We can also confirm this by Theorem 2.8. Let $L=\mathbb{Z}[u, v, w]$ and $K=\mathbb{Z}[u,(v+w) / 3]$ correspond to $16 x^{2}+20 y^{2}+25 z^{2}$ and $\eta$, respectively. It is clear that $K_{p} \subseteq L_{p}$ for $p=2,5$ and $[K: L \cap K]=3 \mathbb{Z}$. Since 3 is inert in $\mathbb{Q}(\sqrt{-1}), \eta$ is not represented by the spinor genus of $16 x^{2}+20 y^{2}+25 z^{2}$.

\section{Representation by spinor genera II, codimension two}

In this section, we assume $\operatorname{rank}(L)-\operatorname{rank}(K)=2$. Write $V=F K \perp W$. It is well-known that every spinor genus in gen $(L)$ represents $K$ unless the following two conditions hold (see [HSX98]):

$$
\operatorname{det}(W) \neq-1
$$




\section{W. K. Chan and F. XU}

and

$$
\theta_{\mathrm{A}}(\operatorname{gen}(L): K)=\theta_{\mathrm{A}}\left(S O_{\mathrm{A}}(W)\right) .
$$

Let $E=F(\sqrt{-\operatorname{det}(W)})$. The following proposition is the analog of Proposition 2.3 in the codimension two case.

Proposition 3.3. Suppose that $L_{\mathfrak{p}}$ and $K_{\mathfrak{p}}$ are unimodular with $\operatorname{rank}\left(L_{\mathfrak{p}}\right) \leqslant 4$ for a non-dyadic prime $\mathfrak{p}$ of $F$. If $\left[K_{\mathfrak{p}}: L_{\mathfrak{p}} \cap K_{\mathfrak{p}}\right]=\mathfrak{p}^{r}$, then $\pi_{\mathfrak{p}} \in \theta_{\mathfrak{p}}\left(X\left(L_{\mathfrak{p}} / K_{\mathfrak{p}}\right)\right)$ if and only if $r$ is odd.

Proof. We only consider the $\operatorname{rank}\left(L_{\mathfrak{p}}\right)=4$ case here and leave the $\operatorname{rank}\left(L_{\mathfrak{p}}\right)=3$ case to the readers. Write $K_{\mathfrak{p}}=\mathfrak{o}_{F_{\mathfrak{p}}} x \perp \mathfrak{o}_{F_{\mathfrak{p}}} y$ with $B(x, L) \mathfrak{o}_{F_{\mathfrak{p}}}=B\left(K_{\mathfrak{p}}, L_{\mathfrak{p}}\right) \mathfrak{o}_{F_{\mathfrak{p}}}=\mathfrak{p}^{-s}$ and $s>0$. Then there is $f \in L_{\mathfrak{p}}$ such that $B(x, f)=\pi_{\mathfrak{p}}^{-s}$ and $Q(f)=0$. Therefore, $L_{\mathfrak{p}}=\left(\mathfrak{p}^{s} x+\mathfrak{o}_{F_{\mathfrak{p}}} f\right) \perp\left(\mathfrak{o}_{F_{\mathfrak{p}}} u+\mathfrak{o}_{F_{\mathfrak{p}}} v\right)$ for some $u$ and $v$ in $L_{\mathfrak{p}}$. If $y=a x+b f+c u+d v$, then $B(y, f) \in \mathfrak{p}^{-s}$ and $B(x, y)=0$. Therefore, $a \in \mathfrak{o}_{F_{\mathfrak{p}}}$ and $b \in \mathfrak{p}^{s}$.

When $F_{\mathfrak{p}} u+F_{\mathfrak{p}} v$ is anisotropic, then $c$ and $d$ are in $\mathfrak{o}_{F_{\mathfrak{p}}}$ because

$$
Q(c u+d v)=Q(y)-Q(a x)-2 a b \pi_{\mathfrak{p}}^{-s} \in \mathfrak{o}_{F_{\mathfrak{p}}} .
$$

Therefore, $\left[K_{\mathfrak{p}}: K_{\mathfrak{p}} \cap L_{\mathfrak{p}}\right]=\mathfrak{p}^{s}$ and $r=s$. Let $\sigma \in S O\left(V_{\mathfrak{p}}\right)$ be defined by

$$
\sigma: \pi_{\mathfrak{p}}^{r}\left(x-\frac{1}{2} Q(x) \pi_{\mathfrak{p}}^{r} f\right) \rightarrow\left(x-\frac{1}{2} Q(x) \pi_{\mathfrak{p}}^{r} f\right), f \rightarrow \pi_{\mathfrak{p}}^{r} f, u \rightarrow u, v \rightarrow v .
$$

Then $X\left(L_{\mathfrak{p}} / K_{\mathfrak{p}}\right)=\sigma S O\left(L_{\mathfrak{p}}\right)$ and $\theta_{\mathfrak{p}}\left(\sigma_{r}\right) \in \pi_{\mathfrak{p}}^{r} \mathfrak{u}_{\mathfrak{p}}\left(F_{\mathfrak{p}}^{\times}\right)^{2}$.

When $F_{\mathfrak{p}} u+F_{\mathfrak{p}} v$ is isotropic, we can assume that $Q(u)=Q(v)=0$ and $B(u, v)=1$. Therefore, $c d \in \mathfrak{o}_{F_{\mathfrak{p}}}$. When both $c$ and $d$ are in $\mathfrak{o}_{F_{\mathfrak{p}}},\left[K_{\mathfrak{p}}: K_{\mathfrak{p}} \cap L_{\mathfrak{p}}\right]=\mathfrak{p}^{s}$ and we are done as in the last paragraph. Therefore, we may assume that $\operatorname{ord}(c)<0$. Then $K_{\mathfrak{p}} \cap L_{\mathfrak{p}}=\mathfrak{p}^{s} x+\mathfrak{p}^{-\operatorname{ord}(c)}(b f+c u+d v)$ and $\left[K_{\mathfrak{p}}: K_{\mathfrak{p}} \cap L_{\mathfrak{p}}\right]=\mathfrak{p}^{s-\operatorname{ord}(c)}$. If we put

$$
\sigma: \pi_{\mathfrak{p}}^{s}\left(x-\frac{1}{2} Q(x) \pi_{\mathfrak{p}}^{s} f\right) \rightarrow\left(x-\frac{1}{2} Q(x) \pi_{\mathfrak{p}}^{s} f\right), f \rightarrow \pi_{\mathfrak{p}}^{s} f, u \rightarrow \pi_{\mathfrak{p}}^{\operatorname{ord}(c)} u, v \rightarrow \pi_{\mathfrak{p}}^{-\operatorname{ord}(c)} v,
$$

then $\sigma \in X\left(L_{\mathfrak{p}} / K_{\mathfrak{p}}\right)=\sigma S O\left(L_{\mathfrak{p}}\right)$ and $\theta_{\mathfrak{p}}(\sigma) \in \pi_{\mathfrak{p}}^{r} \mathfrak{u}_{\mathfrak{p}}\left(F_{\mathfrak{p}}^{\times}\right)^{2}$. The proof is now complete.

Remark 3.4. Proposition 3.3 is not true for dyadic primes. For example, $\theta_{\mathfrak{p}}\left(X\left(L_{\mathfrak{p}} / K_{\mathfrak{p}}\right)\right)=F_{\mathfrak{p}} \times$ when $\operatorname{rank}\left(L_{\mathfrak{p}}\right)=4$ and $\mathfrak{n}\left(K_{\mathfrak{p}}\right)=\mathfrak{n}\left(L_{\mathfrak{p}}\right)$ [Xu99, Reduction Formula III] or $\operatorname{rank}\left(L_{\mathfrak{p}}\right)=3[\mathrm{Xu} 00$, Theorem 2.1].

Let $S_{0}$ be the set containing all finite primes that divide $2 \mathfrak{v}(L)$. By weak approximation, there is $M \in \operatorname{cls}(L)$ such that

$$
K_{\mathfrak{p}} \subset M_{\mathfrak{p}} \text { for all } \mathfrak{p} \in S_{0}
$$

Define

$$
i(M, K)=\prod_{\mathfrak{p}<\infty}\left[K_{\mathfrak{p}}: K_{\mathfrak{p}} \cap M_{\mathfrak{p}}\right]
$$

and

$$
\mathfrak{I}(\operatorname{cls}(L), K)=\{i(M, K): M \in \operatorname{cls}(L) \text { with }(3.5)\} .
$$

Theorem 3.6. Suppose the rank of $L$ is less than or equal to four and (3.1) and (3.2) hold. Let $\mathfrak{a}$ be an ideal in $\mathfrak{I}(\operatorname{cls}(L), K)$. Then $K$ is represented by $\operatorname{spn}(L)$ if and only if $\mathfrak{a}$ is trivial in $\operatorname{Gal}(E / F)$ under the Artin map.

Proof. By (3.1), (3.2) and class field theory, we have $\mathbf{I}_{F} / F^{\times} \theta_{\mathrm{A}}(\operatorname{gen}(L), K) \cong \operatorname{Gal}(E / F)$. It is clear that $\mathfrak{p}$ is unramified in $E / F$ for any finite $\mathfrak{p} \notin S_{0}$.

If $\mathfrak{p} \notin S_{0}$ and $\mathfrak{p} \mid \mathfrak{v}(K)$, then $\theta_{\mathfrak{p}}\left(X\left(L_{\mathfrak{p}} / K_{\mathfrak{p}}\right)\right)=F_{\mathfrak{p}}^{\times}$by [HSX98, Theorem 5.1]. The result follows from Proposition 3.3 and (2.2). 


\section{REPRESENTATIONS OF SPINOR GENERA}

The following proposition subsumes the main theorem in [EH82] as a special case.

Proposition 3.7. Suppose that $L$ is a unimodular lattice of rank 3 and (3.1) and (3.2) hold. If $\mathfrak{b}=B(K, L) \mathfrak{o}_{F}$, then $K$ is represented by $\operatorname{spn}(L)$ if and only if $\mathfrak{b}$ is trivial in $\operatorname{Gal}(E / F)$ under the Artin map.

Proof. It is clear that $S_{0}$ contains only the dyadic primes. Moreover, $\theta_{\mathfrak{p}}\left(X\left(L_{\mathfrak{p}} / K_{\mathfrak{p}}\right)\right)=F_{\mathfrak{p}}^{\times}$for any $\mathfrak{p} \in S_{0}$ by [Xu00, Theorem 2.1]. The proposition now follows from Proposition 3.3, (2.2), and Remark 2.4.

We give one more illustration of Theorem 3.6 by the following example which was also considered in [Bor01] and [BR95].

Example 3.8. Let $L=\mathbb{Z}[u, v, w]$ be an indefinite ternary $\mathbb{Z}$-lattice such that

$$
Q(x u+y v+z w)=-9 x^{2}+2 x y+7 y^{2}+2 z^{2} .
$$

Since $Q\left(\frac{1}{3} u+z\right)=Q\left(-\frac{1}{2} u+\frac{1}{2} v+w\right)=1$, therefore 1 is represented by $\operatorname{gen}(L)$. As $S_{0}=\{2\}$ in this case, we can take $K$ to be the rank 1 lattice spanned by the vector $\frac{1}{3} u+z$. Then $E=\mathbb{Q}(\sqrt{2})$ and $3 \mathbb{Z}$ is in $\mathfrak{I}(\operatorname{cls}(L), K)$. It is clear that 3 is inert in $\mathbb{Q}(\sqrt{2})$. Therefore 1 is not represented by $L$ by Theorem 3.6.

\section{Integral embedding for quaternion algebras}

Let $\mathfrak{B}$ be a quaternion algebra over $F$ and $U$ be a two-dimensional commutative $F$-subalgebra of $\mathfrak{B}$. Let $\mathcal{D}$ be an order in $\mathfrak{B}$ and $\Omega$ be an order in $U$. The set of pure quaternion in $\mathfrak{B}$ is denoted by $\mathfrak{B}^{0}$. For any $S \subseteq \mathfrak{B}, S^{0}$ is the set $S \cap \mathfrak{B}^{0}$. On $\mathfrak{B}$, we have a symmetric bilinear form $(x, y) \longmapsto \mathbb{T}(x \bar{y})$ where $\mathbb{T}$ is the reduced trace from $\mathfrak{B}$ to $F$ and ${ }^{-}$is the main involution on $\mathfrak{B}$. The quadratic map on $\mathfrak{B}$ is $2 \mathbb{N}$, where $\mathbb{N}$ is the reduced norm. As a quadratic space, $U$ can be degenerate. In this case, there is $v \in U$ such that $U=F+F v$ and $\mathbb{T}(v)=0$ and $v^{2}=0$. Then there is $a \in F^{\times}$such that $\Omega \subseteq \mathfrak{o}_{F}+\mathfrak{o}_{F} a v$. It is clear that $\mathfrak{B}$ is split in this case. Then there is a non-zero element $x \in \mathcal{D}$ such that $\mathbb{T}(x)=0$ and $x^{2}=0$. It is easy to see that the map which sends $a v$ to $x$ gives an embedding of $\Omega$ to $\mathcal{D}$. Therefore, we can always assume that $U$ is non-degenerate as a quadratic space.

Let $K$ be the lattice $\Omega^{0}, J=\left[\Omega: \mathfrak{o}_{F} \perp K\right]$ and $L$ be the lattice $\left(\mathfrak{o}_{F}+J \mathcal{D}\right)^{0}$. Note that $J$ is only divisible by the dyadic primes and the norm of $\Omega$ as a quadratic lattice is always $2 \mathfrak{o}_{F}$.

Let $\mathfrak{p}$ be a dyadic prime, $\Delta=1-4 \rho$ be a unit of quadratic defect $4 \mathfrak{o}_{F_{\mathfrak{p}}}$ and $e=\operatorname{ord}(2)$. Then $\Omega_{\mathfrak{p}}$ is not split by $\mathfrak{o}_{F_{\mathfrak{p}}}$ if and only if

$$
\Omega_{\mathfrak{p}}=\mathfrak{o}_{F_{\mathfrak{p}}}+\mathfrak{o}_{F_{\mathfrak{p}}} u_{\mathfrak{p}} \quad \text { where } \mathbb{T}\left(u_{\mathfrak{p}}\right)=\pi_{\mathfrak{p}}^{r_{\mathfrak{p}}} \text { and } r_{\mathfrak{p}}<e .
$$

In this case, $J_{\mathfrak{p}}=\mathfrak{p}^{s_{\mathfrak{p}}}$ with $s_{\mathfrak{p}}=e-r_{\mathfrak{p}}$ and $K_{\mathfrak{p}}=\mathfrak{o}_{F_{\mathfrak{p}}} v_{\mathfrak{p}}$ with $\mathbb{N}\left(v_{\mathfrak{p}}\right)=\pi_{\mathfrak{p}}^{-2 r_{\mathfrak{p}}} \operatorname{det}\left(\Omega_{\mathfrak{p}}\right)$ and

$$
v_{\mathfrak{p}}=-1+2 \pi_{\mathfrak{p}}^{-r_{\mathfrak{p}}} u_{\mathfrak{p}}
$$

Proposition 4.3. $\Omega \subseteq \mathcal{D}$ if and only if $K \subseteq L$.

Proof. Suppose $\Omega \subseteq \mathcal{D}$. If $\mathfrak{p}$ does not divide $J$, then $\Omega_{\mathfrak{p}}=\mathfrak{o}_{F_{\mathfrak{p}}} \perp K_{\mathfrak{p}}$ and $L_{\mathfrak{p}}=\mathcal{D}_{\mathfrak{p}}^{0}$. Therefore, $K_{\mathfrak{p}} \subseteq L_{\mathfrak{p}}$. If $\mathfrak{p} \mid J$, then by $(4.2)$ we know that $v_{\mathfrak{p}} \in \mathfrak{o}_{F_{\mathfrak{p}}}+J_{\mathfrak{p}} \mathcal{D}_{\mathfrak{p}}$ and hence $K_{\mathfrak{p}} \subseteq L_{\mathfrak{p}}$.

Conversely, suppose $K \subseteq L$. It is clear that $\Omega_{\mathfrak{p}} \subseteq \mathcal{D}_{\mathfrak{p}}$ for all $\mathfrak{p} \nmid J$. For $\mathfrak{p} \mid J$, we only need to show that $\left(1+v_{\mathfrak{p}}\right) / \pi_{\mathfrak{p}}^{s_{\mathfrak{p}}}$ is in $\mathcal{D}_{\mathfrak{p}}$ by (4.2). Since $v_{\mathfrak{p}} \in L_{\mathfrak{p}}$, we can write $v_{\mathfrak{p}}=A+\pi_{\mathfrak{p}}^{s_{\mathfrak{p}}} w$ where $A \in \mathfrak{o}_{F_{\mathfrak{p}}}$ and $w \in \mathcal{D}_{\mathfrak{p}} \cap U_{\mathfrak{p}}$. Therefore, $(1+A) / \pi_{\mathfrak{p}}^{s_{\mathfrak{p}}}=\epsilon_{1} u_{\mathfrak{p}}-w$ is an integral element in $U_{\mathfrak{p}}$. Hence

$$
\frac{1+v_{\mathfrak{p}}}{\pi_{\mathfrak{p}}^{S_{\mathfrak{p}}}}=\frac{1+A}{\pi_{\mathfrak{p}}^{s_{\mathfrak{p}}}}+w \in \mathcal{D}_{\mathfrak{p}}
$$

and this implies $\Omega_{\mathfrak{p}} \subseteq \mathcal{D}_{\mathfrak{p}}$. 


\section{W. K. Chan AND F. XU}

Remark 4.4. In general, the sum of two integral quaternions may not be integral. However, if these two quaternions commute with each other, then their sum is integral.

When $\mathfrak{B}^{0}$ is regarded as a quadratic space, the proper isometries are induced from the conjugation of elements of $\mathfrak{B}^{\times}$. Let gen $(\mathcal{D})$ be the orders in $\mathfrak{B}$ that are conjugate to $\mathcal{D}$ at every local completion of $F$. For any order $\mathcal{E}$ in $\operatorname{gen}(\mathcal{D})$, let $\Phi(\mathcal{E})$ be the lattice $\left(\mathfrak{o}_{F}+J \mathcal{E}\right)^{0}$. We always assume that $\mathfrak{B}$ satisfies the Eichler condition. By virtue of the strong approximation theorem, both the conjugacy classes in gen $(\mathcal{D})$ and the proper classes in gen $(L)$ have natural 2-elementary abelian group structures and $\Phi$ induces a surjective group homomorphism between them. By Proposition $4.3, K$ is represented by only half of the classes in gen $(L)$ (i.e. $K$ is an exceptional lattice for gen $(L)$ ) if and only if $\Omega$ can be embedded in only half of the conjugacy classes in $\operatorname{gen}(\mathcal{D})$.

By the above observation, it is clear that the results in [CF99], for example Theorem 3.3, can be extended to arbitrary orders since the exceptional lattices have been characterized in [Sch80], [HSX98] and [Xu00]. To explain this, we give an alternative proof of [CF99, Theorem 3.3] via this approach. Write $\mathfrak{B}^{0}=F K \perp W$ as a quadratic space. Then $\mathfrak{B}=U \perp W$. For a maximal order $\mathcal{D}$, one has

$$
\mathcal{D}_{\mathfrak{p}} \cong \begin{cases}A(2,0) \perp A(0,0) & \text { when } \mathfrak{B}_{\mathfrak{p}} \text { is unramified } \\ A(2,2 \rho) \perp \pi_{\mathfrak{p}} A(2,2 \rho) & \text { when } \mathfrak{B}_{\mathfrak{p}} \text { is ramified }\end{cases}
$$

We also have

$$
2 \mathfrak{n}(K)=J^{2} d_{\Omega / \mathfrak{o}_{F}} .
$$

As we pointed out in $\S 3, K$ is an exceptional lattice for gen $(L)$ if and only if $(3.1)$ and $(3.2)$ hold. We are going to show that (3.1) and (3.2) are equivalent to the following three conditions in [CF99, Theorem 3.3]:

1) $\Omega$ is an integral domain and $U$ is a quadratic extension of $F$;

2) the extension $U / F$ and the algebra $\mathfrak{B}$ are unramified at all finite places and ramify at exactly the same (possibly) set of real places of $F$;

3) all prime ideals of $F$ dividing $d_{\Omega / \mathfrak{o}}$ are split in $U / F$.

Without loss of generality, we assume that $\Omega \subset \mathcal{D}$. It is clear that (3.1) is equivalent to condition 1 and $U=F(\sqrt{-\operatorname{det} W})$.

Suppose (3.2) holds. Since $\theta_{\mathfrak{p}}\left(S O\left(L_{\mathfrak{p}}\right)\right) \supseteq \mathfrak{u}_{\mathfrak{p}}$ for all $\mathfrak{p}<\infty$ by (4.5), $U / F$ is unramified for all $\mathfrak{p}<\infty$. If $\mathfrak{B}_{\mathfrak{p}}$ is ramified, then $\mathfrak{p}$ has to be inert in $U / F$ and $\theta_{\mathfrak{p}}\left(S O\left(L_{\mathfrak{p}}\right)\right)=F_{\mathfrak{p}}^{\times}$by $(4.5)$. A contradiction is derived by (3.2). Therefore condition 2 holds for $\mathfrak{p}<\infty$.

When $\mathfrak{p} \mid d_{\Omega / \mathfrak{o}_{F}}$, then $\theta_{\mathfrak{p}}\left(X\left(L_{\mathfrak{p}} / K_{\mathfrak{p}}\right)\right)=F_{\mathfrak{p}}^{\times}$by (4.6) and [HSX98, Theorem 5.1] and [Xu00, Theorem 2.0]. Therefore condition 3 also follows from (3.2).

Conversely, suppose conditions 2 and 3 hold. Since (3.2) is always true if $\mathfrak{p}$ is split in $U / F$, we only need to prove (3.2) for an inert prime $\mathfrak{p}$. Then $\mathfrak{p} \nmid d_{\Omega / \mathfrak{o}_{F \mathfrak{p}}}$ by condition 3 .

If $\mathfrak{p}$ is non-dyadic, $L_{\mathfrak{p}}$ is unimodular and $\theta_{\mathfrak{p}}\left(S O\left(L_{\mathfrak{p}}\right)\right)=\mathfrak{u}_{\mathfrak{p}}$ by condition 2 and (4.5). It is clear that

$$
X\left(L_{\mathfrak{p}} / K_{\mathfrak{p}}\right)=S O\left(L_{\mathfrak{p}}\right) S O\left(W_{\mathfrak{p}}\right)
$$

by (4.6). Therefore (3.2) follows from condition 2 .

If $\mathfrak{p}$ is dyadic prime, then $J=2 \mathfrak{o}_{F_{\mathfrak{p}}}$ by (4.1) and $K \cong\langle-2 \Delta\rangle$ by (4.2) and $L_{\mathfrak{p}} \cong\langle-2 \Delta\rangle \perp$ $4 A(2,2 \rho)$ by condition 2 and (4.5). By [Xu00, Theorem 2.1, Case III(ii)], (3.2) also holds in this case by condition 2 .

For $\mathfrak{p} \mid \infty$, it is clear that $X_{\mathfrak{p}}\left(L_{\mathfrak{p}} / K_{\mathfrak{p}}\right)=S O\left(\mathfrak{B}_{\mathfrak{p}}^{0}\right)$. Therefore, (3.2) at $\mathfrak{p}$ is equivalent to the statement that $\mathfrak{B}_{\mathfrak{p}}$ is ramified if and only if $U / F$ is ramified at $\mathfrak{p}$. 


\section{REPRESENTATIONS OF SPINOR GENERA}

Suppose that conditions 1,2 and 3 hold and $\Omega \subset \mathcal{D}$. Let $\mathcal{E}$ be another maximal order in $\mathfrak{B}$ and $M=\left(\mathfrak{o}_{F}+J \mathcal{E}\right)^{0}$. In $\S 2$, we point out that $K$ is represented by $M$ if and only if (2.2) holds. It is clear that we only need to consider those $\mathfrak{p}$ which are inert in $U / F$. For such a $\mathfrak{p}$, we can write

$$
\mathcal{D}_{\mathfrak{p}}=\left(\mathfrak{o}_{F_{\mathfrak{p}}}+\mathfrak{o}_{F_{\mathfrak{p}}} x\right) \perp\left(\mathfrak{o}_{F_{\mathfrak{p}}} y+\mathfrak{o}_{F_{\mathfrak{p}}} z\right) \quad \text { and } \quad \mathcal{E}_{\mathfrak{p}}=\left(\mathfrak{o}_{F_{\mathfrak{p}}}+\mathfrak{o}_{F_{\mathfrak{p}}} x\right) \perp\left(\mathfrak{p}^{n} y+\mathfrak{p}^{-n} z\right)
$$

where $\mathbb{N}(x)=\mathbb{N}(y)=\mathbb{N}(z)=0$ and $\mathbb{T}(x)=\mathbb{T}(y \bar{z})=1$.

If $\mathfrak{p}$ is non-dyadic, then $\mathcal{E}_{\mathfrak{p}}$ and $\mathcal{D}_{\mathfrak{p}}$ are split by $\mathfrak{o}_{F_{\mathfrak{p}}}$. Therefore, $\left[\mathcal{E}_{\mathfrak{p}}: \mathcal{E}_{\mathfrak{p}} \cap \mathcal{D}_{\mathfrak{p}}\right]=\left[M_{\mathfrak{p}}: L_{\mathfrak{p}} \cap M_{\mathfrak{p}}\right]=\mathfrak{p}^{n}$.

If $\mathfrak{p}$ is dyadic, then $\mathfrak{p} \nmid d_{\Omega / \mathfrak{o}_{F}}$ by condition 3. By (4.1), we have $J_{\mathfrak{p}}=2 \mathfrak{o}_{F_{\mathfrak{p}}}$ and

$$
L_{\mathfrak{p}}=\mathfrak{o}_{F_{\mathfrak{p}}}(2 x-1) \perp\left(\mathfrak{o}_{F_{\mathfrak{p}}} 2 y+\mathfrak{o}_{F_{\mathfrak{p}}} 2 z\right) \quad \text { and } \quad M_{\mathfrak{p}}=\mathfrak{o}_{F_{\mathfrak{p}}}(2 x-1) \perp\left(\mathfrak{p}^{n} 2 y+\mathfrak{p}^{-n} 2 z\right)
$$

and hence $\left[\mathcal{E}_{\mathfrak{p}}: \mathcal{E}_{\mathfrak{p}} \cap \mathcal{D}_{\mathfrak{p}}\right]=\left[M_{\mathfrak{p}}: L_{\mathfrak{p}} \cap M_{\mathfrak{p}}\right]=\mathfrak{p}^{n}$. It is clear that $\pi_{\mathfrak{p}} \in \theta_{\mathfrak{p}}\left(X\left(M_{\mathfrak{p}} / K_{\mathfrak{p}}\right)\right)$ if and only if $n$ is odd. Therefore, $K$ is represented by $M$ if and only if the distance ideal $\rho(\mathcal{D}, \mathcal{E})$ is trivial in $\operatorname{Gal}(U / F)$ (see also [HSX98, Theorem 4.1]).

Based on the above arguments, we extend Theorem 3.3 in [CF99] to Eichler orders of level $\mathfrak{d}$.

Theorem 4.7. Suppose that $\mathcal{D}$ is an Eichler order in $\mathfrak{B}$ of level $\mathfrak{d}$ and $\Omega$ is an order in $U$. Assume that $\Omega$ is embedded into a conjugacy class in $\operatorname{gen}(\mathcal{D})$. Then $\Omega$ is embedded into either all or exactly half of the conjugacy classes in gen $(\mathcal{D})$, and the latter happens if and only if conditions 1 and 2 and the following condition are satisfied:

$\left.3^{\prime}\right)$ All prime ideals of $F$ dividing $d_{\Omega / \mathfrak{o}_{F}}$ are split in $U / F$ unless $\operatorname{ord}_{\mathfrak{p}}(\mathfrak{d})=\operatorname{ord}_{\mathfrak{p}}\left(d_{\Omega / \mathfrak{o}_{F}}\right)$.

Suppose that conditions 1,2 and $3^{\prime}$ hold. Then $\Omega$ is embedded into $\mathcal{D}$ if and only if $[\Omega: \Omega \cap \mathcal{D}]$ is trivial in $\operatorname{Gal}(U / F)$ under the Artin map.

Proof. It is clear that condition 1 is equivalent to (3.1) and we only need to consider $\mathfrak{p} \mid \mathfrak{d}$ by the above argument. For such a $\mathfrak{p}, \mathfrak{B}_{\mathfrak{p}}$ must be unramified. Let $a_{\mathfrak{p}}=\operatorname{ord} \mathrm{d}_{\mathfrak{p}}(\mathfrak{d})$ and $d_{\mathfrak{p}}=\operatorname{ord}_{\mathfrak{p}}\left(d_{\Omega / \mathfrak{o}_{F}}\right)$. For the first part of the theorem, we may assume that $K_{\mathfrak{p}} \subset L_{\mathfrak{p}}$ for all $\mathfrak{p}$.

Non-dyadic prime $\mathfrak{p}$. We have $L_{\mathfrak{p}} \cong\langle-2\rangle \perp \pi^{a_{\mathfrak{p}}} A(0,0)$ and $K_{\mathfrak{p}} \cong\left\langle 2 \operatorname{det}\left(\Omega_{\mathfrak{p}}\right)\right\rangle$. Since $K_{\mathfrak{p}}$ is represented by $L_{\mathfrak{p}}$, we also have $d_{\mathfrak{p}} \geqslant a_{\mathfrak{p}}$ when $\mathfrak{p}$ is inert. So by [HSX98, Theorem 5.1]

$$
\theta_{\mathfrak{p}}\left(X\left(L_{\mathfrak{p}} / K_{\mathfrak{p}}\right)\right)= \begin{cases}\mathfrak{u}_{\mathfrak{p}} F_{\mathfrak{p}}^{\times} & \text {if } a_{\mathfrak{p}}=d_{\mathfrak{p}} \text { and } \mathfrak{p} \text { is inert } \\ F_{\mathfrak{p}}^{\times} & \text {otherwise. }\end{cases}
$$

It is clear that (3.2) is equivalent to conditions 2 and $3^{\prime}$.

Dyadic prime $\mathfrak{p}$. Since $\theta_{\mathfrak{p}}\left(S O\left(L_{\mathfrak{p}}\right)\right) \supseteq \mathfrak{u}_{\mathfrak{p}}$, it is clear that $\theta_{\mathfrak{p}}\left(X\left(L_{\mathfrak{p}} / K_{\mathfrak{p}}\right)\right)=F_{\mathfrak{p}}^{\times}$when $\mathfrak{p}$ is not inert in $U / F$. Let $\mathfrak{p}$ be an inert prime. Then $\operatorname{det}\left(\Omega_{\mathfrak{p}}\right)=-\Delta \pi^{d_{\mathfrak{p}}}$ where $d_{\mathfrak{p}}$ is even. Let $e=\operatorname{ord}_{\mathfrak{p}}(2)$.

If $\mathfrak{p} \mid J$, then $L_{\mathfrak{p}} \cong\langle-2\rangle \perp \pi^{a_{\mathfrak{p}}+2 s_{\mathfrak{p}}} A(0,0)$ and $K_{\mathfrak{p}} \cong\langle-2 \Delta\rangle$. Since $K_{\mathfrak{p}}$ is represented by $L_{\mathfrak{p}}$, we have $a_{\mathfrak{p}}+2 s_{\mathfrak{p}}=a_{\mathfrak{p}}+2 e-d_{\mathfrak{p}} \leqslant 2 e$ by (4.5) and so $d_{\mathfrak{p}} \geqslant a_{\mathfrak{p}}$. By [Xu00, Theorem 2.1, Case III],

$$
\theta_{\mathfrak{p}}\left(X\left(L_{\mathfrak{p}} / K_{\mathfrak{p}}\right)\right)= \begin{cases}\mathfrak{u}_{\mathfrak{p}} F_{\mathfrak{p}}^{\times 2} & \text { if } a_{\mathfrak{p}}=d_{\mathfrak{p}} \text { and } \mathfrak{p} \text { is inert } \\ F_{\mathfrak{p}}^{\times} & \text {otherwise. }\end{cases}
$$

Therefore (3.2) is equivalent to conditions 2 and $3^{\prime}$.

If $\mathfrak{p} \nmid J$, then $L_{\mathfrak{p}} \cong\langle-2\rangle \perp \pi^{a_{\mathfrak{p}}} A(0,0)$ and $K_{\mathfrak{p}} \cong\left\langle-2^{-1} \pi^{d_{\mathfrak{p}}} \Delta\right\rangle$. Since $\mathfrak{n}\left(K_{\mathfrak{p}}\right) \subseteq \mathfrak{n}\left(L_{\mathfrak{p}}\right), d_{\mathfrak{p}} \geqslant 2 e$.

When $d_{\mathfrak{p}}-2 e \geqslant a_{\mathfrak{p}}$, then $\theta_{\mathfrak{p}}\left(X\left(L_{\mathfrak{p}} / K_{\mathfrak{p}}\right)\right)=F_{\mathfrak{p}}^{\times}$by [Xu00, Theorem 2.0]. 


\section{W. K. Chan AND F. XU}

When $d_{\mathfrak{p}}-2 e<a_{\mathfrak{p}}$, then $a_{\mathfrak{p}}-\left(d_{\mathfrak{p}}-2 e\right) \leqslant 2 e$ because $K_{\mathfrak{p}}$ is represented by $L_{\mathfrak{p}}$. Thus $a_{\mathfrak{p}} \leqslant d_{\mathfrak{p}}$. By [Xu00, Theorem 2.1, Case III],

$$
\theta_{\mathfrak{p}}\left(X\left(L_{\mathfrak{p}} / K_{\mathfrak{p}}\right)\right)= \begin{cases}\mathfrak{u}_{\mathfrak{p}} F_{\mathfrak{p}}^{\times 2} & \text { if } a_{\mathfrak{p}}=d_{\mathfrak{p}} \text { and } \mathfrak{p} \text { is inert } \\ F_{\mathfrak{p}}^{\times} & \text {otherwise. }\end{cases}
$$

Therefore (3.2) is equivalent to conditions 2 and $3^{\prime}$.

Now we prove the second part of the theorem. First we claim that $[\Omega: \Omega \cap \mathcal{D}]=[K: K \cap L]$.

If $\mathfrak{p}$ is non-dyadic or dyadic with $\mathfrak{p} \nmid J$, then $\Omega_{\mathfrak{p}}=\mathfrak{o}_{F_{\mathfrak{p}}} \perp K_{\mathfrak{p}}$ and $\mathfrak{o}_{F_{\mathfrak{p}}} \perp\left(\mathcal{D}_{\mathfrak{p}} \cap K_{\mathfrak{p}}\right)=$ $\mathcal{D}_{\mathfrak{p}} \cap\left(\mathfrak{o}_{F_{\mathfrak{p}}} \perp K_{\mathfrak{p}}\right)$. Therefore, $\left[\Omega_{\mathfrak{p}}: \Omega_{\mathfrak{p}} \cap \mathcal{D}_{\mathfrak{p}}\right]=\left[\mathfrak{o}_{F_{\mathfrak{p}}} \perp K_{\mathfrak{p}}:\left(\mathfrak{o}_{F_{\mathfrak{p}}} \perp K_{\mathfrak{p}}\right) \cap \mathcal{D}_{\mathfrak{p}}\right]=\left[K_{\mathfrak{p}}: K_{\mathfrak{p}} \cap \mathcal{D}_{\mathfrak{p}}\right]=\left[K_{\mathfrak{p}}:\right.$ $\left.K_{\mathfrak{p}} \cap L_{\mathfrak{p}}\right]$.

If $\mathfrak{p}$ is dyadic and $\mathfrak{p} \mid J$, we write

$$
\mathcal{D}_{\mathfrak{p}}=\left(\mathfrak{o}_{F_{\mathfrak{p}}}+\mathfrak{o}_{F_{\mathfrak{p}}} x\right) \perp\left(\mathfrak{o}_{F_{\mathfrak{p}}} y+\mathfrak{o}_{F_{\mathfrak{p}}} z\right) \cong A(2,0) \perp \pi^{a_{\mathfrak{p}}} A(0,0) .
$$

Let $k$ be the smallest integer such that $\pi_{\mathfrak{p}}^{k} u_{\mathfrak{p}} \in \mathcal{D}_{\mathfrak{p}}$, where $u_{\mathfrak{p}}$ is the same as that in (4.1). Then $\left[\Omega_{\mathfrak{p}}\right.$ : $\left.\Omega_{\mathfrak{p}} \cap \mathcal{D}_{\mathfrak{p}}\right]=\mathfrak{p}^{k}$. By Proposition 4.3, we can assume that $k \geqslant 1$. Let $\pi^{k} u_{\mathfrak{p}}=a+b x+c y+d z$, where $a, b, c$ and $d$ are in $\mathfrak{o}_{F_{\mathfrak{p}}}$ and one of them is a unit. By taking the trace and the norm, we have $\pi_{\mathfrak{p}}^{k+r_{\mathfrak{p}}}=2 a+b$ and $\pi_{\mathfrak{p}}^{2 k} \mathbb{N}\left(u_{\mathfrak{p}}\right)=a^{2}+a b+c d \mathbb{T}(y \bar{z})$. It is clear that $b$ is not a unit and one of $c$ and $d$ has to be a unit. Since $L_{\mathfrak{p}}=\mathfrak{o}_{F_{\mathfrak{p}}}(2 x-1) \perp\left(\mathfrak{p}^{e-r_{\mathfrak{p}}} y+\mathfrak{p}^{e-r_{\mathfrak{p}}} z\right), K_{\mathfrak{p}}=\mathfrak{o}_{F_{\mathfrak{p}}}\left(2 \pi^{-r_{\mathfrak{p}}} u_{\mathfrak{p}}-1\right)$ and

$$
\pi_{\mathfrak{p}}^{k}\left(2 \pi_{\mathfrak{p}}^{-r_{\mathfrak{p}}} u_{\mathfrak{p}}-1\right)=\pi^{-r_{\mathfrak{p}}} b(2 x-1)+2 \pi_{\mathfrak{p}}^{-r_{\mathfrak{p}}} c y+2 \pi_{\mathfrak{p}}^{-r_{\mathfrak{p}}} d z
$$

we have $\left[K_{\mathfrak{p}}: K_{\mathfrak{p}} \cap L_{\mathfrak{p}}\right]=\mathfrak{p}^{k}$.

For the rest of the proof, it suffices to show the following.

Claim. Let $\mathfrak{p}$ be inert in $U / F$ and $\left[K_{\mathfrak{p}}: K_{\mathfrak{p}} \cap L_{\mathfrak{p}}\right]=\mathfrak{p}^{k}$. Then $\pi_{\mathfrak{p}} \in \theta_{\mathfrak{p}}\left(X\left(L_{\mathfrak{p}} / K_{\mathfrak{p}}\right)\right)$ if and only if $k$ is odd.

For such a prime $\mathfrak{p}$, we can write $\operatorname{det}\left(\Omega_{\mathfrak{p}}\right)=-\varepsilon_{\mathfrak{p}} \pi^{d_{\mathfrak{p}}}$ where $\varepsilon_{\mathfrak{p}}$ is a non-square unit, $d_{\mathfrak{p}}$ is even and $d_{\mathfrak{p}}=a_{\mathfrak{p}}$ by condition $3^{\prime}$. Write

$$
L_{\mathfrak{p}}=\mathfrak{o}_{F_{\mathfrak{p}}} x \perp\left(\mathfrak{o}_{F_{\mathfrak{p}}} y+\mathfrak{o}_{F_{\mathfrak{p}}} z\right) \cong\langle-2\rangle \perp \pi^{a_{\mathfrak{p}}+2 s_{\mathfrak{p}}} A(0,0)
$$

and $K_{\mathfrak{p}}=\mathfrak{o}_{F_{\mathfrak{p}}} v_{\mathfrak{p}}$.

Non-dyadic prime $\mathfrak{p}$. By Proposition 3.3, we may assume that $a_{\mathfrak{p}}=d_{\mathfrak{p}}>0$. It is clear that $X\left(L_{\mathfrak{p}} / K_{\mathfrak{p}}\right)=X\left(L_{\mathfrak{p}}^{\prime} / K_{\mathfrak{p}}\right)$ and $\left[K_{\mathfrak{p}}: K_{\mathfrak{p}} \cap L_{\mathfrak{p}}\right]=\left[K_{\mathfrak{p}}: K_{\mathfrak{p}} \cap L_{\mathfrak{p}}^{\prime}\right]$, where $L_{\mathfrak{p}}^{\prime}=\mathfrak{p}^{d_{\mathfrak{p}} / 2} x \perp\left(\mathfrak{o}_{F_{\mathfrak{p}}} y+\mathfrak{o}_{F_{\mathfrak{p}}} z\right)$. By scaling and Proposition 3.3, $\pi_{\mathfrak{p}} \in \theta_{\mathfrak{p}}\left(X\left(L_{\mathfrak{p}} / K_{\mathfrak{p}}\right)\right)$ if and only if $k$ is odd.

To prove the dyadic cases, we need the following lemma whose proof will be given later.

Lemma 4.8. Let $\mathfrak{p}$ be a dyadic prime. Suppose that

$$
M=\mathfrak{o}_{F_{\mathfrak{p}}} f \perp\left(\mathfrak{o}_{F_{\mathfrak{p}}} g+\mathfrak{o}_{F_{\mathfrak{p}}} h\right) \cong\langle 1\rangle \perp 2 A(0,0) \text { and } \quad N=\mathfrak{o}_{F_{\mathfrak{p}}} l \cong\langle\Delta\rangle
$$

and $[N: M \cap N]=\mathfrak{p}^{j}$. Then $j$ is odd if and only if $\pi \in \theta_{\mathfrak{p}}(X(M / N))$.

Dyadic prime $\mathfrak{p}$. If $\mathfrak{p} \mid J$, then $K_{\mathfrak{p}} \cong\langle-2 \Delta\rangle$ and $a_{\mathfrak{p}}=d_{\mathfrak{p}}=2 r_{\mathfrak{p}}$. By scaling and Lemma 4.8, $\pi_{\mathfrak{p}} \in \theta_{\mathfrak{p}}\left(X\left(L_{\mathfrak{p}} / K_{\mathfrak{p}}\right)\right)$ if and only if $k$ is odd.

If $\mathfrak{p} \nmid J$, then $K_{\mathfrak{p}} \cong\left\langle-2^{-1} \Delta \pi_{\mathfrak{p}}^{d_{\mathfrak{p}}}\right\rangle$ and $d_{\mathfrak{p}}=a_{\mathfrak{p}} \geqslant 2 e$. It is clear that $X\left(L_{\mathfrak{p}} / K_{\mathfrak{p}}\right)=X\left(L_{\mathfrak{p}}^{\prime} / K_{\mathfrak{p}}\right)$ and $\left[K_{\mathfrak{p}}: K_{\mathfrak{p}} \cap L_{\mathfrak{p}}\right]=\left[K_{\mathfrak{p}}: K_{\mathfrak{p}} \cap L_{\mathfrak{p}}^{\prime}\right]$, where, $L_{\mathfrak{p}}^{\prime}=\mathfrak{p}^{d_{\mathfrak{p}} / 2-e} x \perp\left(\mathfrak{o}_{F_{\mathfrak{p}}} y+\mathfrak{o}_{F_{\mathfrak{p}}} z\right)$. By scaling and Lemma 4.8 , $\pi_{\mathfrak{p}} \in \theta_{\mathfrak{p}}\left(X\left(L_{\mathfrak{p}} / K_{\mathfrak{p}}\right)\right)$ if and only if $k$ is odd.

The proof is completed by (2.2) and (3.2). 


\section{REPRESENTATIONS OF SPINOR GENERA}

Proof of Lemma 4.8. When $j=0, N \subseteq M$ and $\theta_{\mathfrak{p}}(X(M / N))=\mathfrak{u}_{\mathfrak{p}} F_{\mathfrak{p}}^{\times 2}$. Therefore, we assume henceforth that $j \geqslant 1$. Since $[N: N \cap M]=\mathfrak{p}^{j}$, there are $\alpha, \beta$ and $\gamma$ in $\mathfrak{o}_{F_{\mathfrak{p}}}$; one of them is a unit, such that $\pi_{\mathfrak{p}}^{j} l=\alpha f+\beta g+\gamma h$. Let $w=x+\rho \xi y-\xi^{-1} z$, where $\xi$ is a unit which will be chosen afterwards. It is clear that $\tau_{l-w} \in X(M / N)$. Also, $\pi_{\mathfrak{p}}^{2 j} \Delta=\alpha^{2}+4 \beta \gamma$ and $Q(l-w)=$ $2\left(\Delta-\alpha \pi_{\mathfrak{p}}^{-j}-2 \rho \xi \gamma \pi_{\mathfrak{p}}^{-j}+2 \beta \xi^{-1} \pi_{\mathfrak{p}}^{-j}\right)$. Therefore, $\operatorname{ord}(\alpha) \geqslant 1$. Without loss of generality, one can assume that $\beta$ is a unit.

Case 1: $j<e$. Then $\operatorname{ord}(\alpha)=j$. Since $\Delta=1-4 \rho, 1-\alpha^{2} \pi_{\mathfrak{p}}^{-2 j}=4 \beta \gamma \pi_{\mathfrak{p}}^{-2 j}+4 \rho$. If $\operatorname{ord}\left(\alpha \pi_{\mathfrak{p}}^{-j}-1\right)$ $<e$, then

$$
\operatorname{ord}\left(\alpha \pi_{\mathfrak{p}}^{-j}+1\right)=\operatorname{ord}\left(\alpha \pi_{\mathfrak{p}}^{-j}-1\right)=e-j+\frac{\operatorname{ord}(\gamma)}{2} .
$$

By a suitable choice of $\xi$, we have ord $(Q(l-w))=2 e-j$.

If $\operatorname{ord}\left(\alpha \pi_{\mathfrak{p}}^{-j}-1\right) \geqslant e$, then $\operatorname{ord}\left(\alpha \pi_{\mathfrak{p}}^{-j}+1\right) \geqslant e$ and $\operatorname{ord}(\gamma) \geqslant 2 j$. Therefore, $\operatorname{ord}(Q(l-w))=$ $e+\operatorname{ord}\left(2 \beta \xi^{-1} \pi_{\mathfrak{p}}^{-j}\right)=2 e-j$.

Case 2: $j=e$. Then $\operatorname{ord}(\alpha) \geqslant e$.

If $\operatorname{ord}(\alpha)=e$, then $\operatorname{ord}(Q(l-w))=e$ by the same argument as that in Case 1.

If $\operatorname{ord}(\alpha)>e$, then both $\beta$ and $\gamma$ are units. It is clear that there is a unit $\xi$ which makes $\rho \xi \gamma-\beta \xi^{-1} \in \mathfrak{p}$. Therefore, $\operatorname{ord}(Q(l-w))=e$.

Case 3: $j>e$. Then $\operatorname{ord}(\alpha) \geqslant e$ and $Q(l-w)=4 \pi_{\mathfrak{p}}^{-j}\left(2^{-1} \pi_{\mathfrak{p}}^{j} \Delta-2^{-1} \alpha-\rho \gamma \xi+\beta \xi^{-1}\right)$.

If $\operatorname{ord}(\alpha)=e$, then both $\beta$ and $\gamma$ are units. We can choose a unit $\xi$ such that $\rho \xi \gamma-\beta \xi^{-1} \in \mathfrak{p}$. Therefore, $\operatorname{ord}(Q(l-w))=2 e-j$.

If $\operatorname{ord}(\alpha)>e$, then $\operatorname{ord}(\gamma) \geqslant 1$. Therefore, $\operatorname{ord}(Q(l-w))=2 e-j$.

Since $X(M / N)=\tau_{l-w} S O(M), \pi_{\mathfrak{p}} \in \theta_{\mathfrak{p}}(X(M / N))$ if and only if $j$ is odd.

Remark 4.9. It is clear that the statement in the second part of Theorem 4.7 is more natural than that in [CF99, Theorem 3.3].

\section{Hardy-Littlewood varieties for indefinite forms}

In this section, we explain how the results in [Xu01] are related to those in [BR95] and answer Question 0.3 in [Bor01].

Let $V$ be a non-degenerate indefinite quadratic space of dimension $n \geqslant 3$ over $\mathbb{Q}, L$ be a $\mathbb{Z}$ lattice with $\mathbb{Q} L=V$, and $K$ be a $\mathbb{Z}$ lattice in $V$ of rank $m$. Let $\mathfrak{L}$ and $\mathfrak{K}$ be the Gram matrices for $L$ and $K$ with respect to some bases. Then we have an affine variety $\mathfrak{Y}$ over $\mathbb{Q}$ defined by $Y \mathfrak{L} Y^{t}=\mathfrak{K}$. The main concern in [BR95] is to determine:

i) if $\mathfrak{Y}$ is strongly Hardy-Littlewood; and

ii) the density function on $\mathfrak{Y}\left(\mathbf{A}_{\mathbb{Q}}\right)$ if $\mathfrak{Y}$ is only Hardy-Littlewood.

Let $N(T, \mathfrak{Y})=\{Y \in \mathfrak{Y}(\mathbb{Z}):|Y| \leqslant T\}$ and $\mu_{\infty}(T, \mathfrak{Y})$ be the singular integral [BR95, (0.0.4)]. We assume that the asymptotic count for $N(T, \mathfrak{Y})$ in [BR95, (0.2.2)] holds in the subsequent discussion. That assumption is known to be valid for $m=1$ and $n \geqslant 3$ (i.e. when $\mathfrak{Y}$ is a symmetric space [DRS93] and [EM93]), and for some other values of $n$ and $m$ [EMS96]. Under $(0.2 .2), \mu_{\infty}(T, \mathfrak{Y})$ is the volume of the set $\{Y \in \mathfrak{Y}(\mathbb{R}):|Y| \leqslant T\}$ by the Tamagawa measure $\bar{\lambda}_{\infty}$ [BR95, Lemma 1.8.2] and

$$
\lim _{T \rightarrow \infty} \frac{N(T, \mathfrak{Y})}{\mu_{\infty}(T, \mathfrak{Y})}=\sum_{\sigma_{\mathrm{A}}} \frac{\mu_{\infty}\left(S O_{\infty}(W) / S O(W) \cap S O\left(\sigma_{\mathrm{A}} L\right)\right)}{\lambda_{\infty}\left(S O_{\infty}(V) / S O(L)\right)}
$$




\section{W. K. Chan AND F. XU}

where $V=\mathbb{Q} K \perp W$ and $\sigma_{\mathrm{A}}$ runs through a complete set of double coset representatives in $S O(W) \backslash X(L / K) / S O_{\mathrm{A}}(L)$ with $\sigma_{\mathrm{A}} L \in \operatorname{cls}(L)$. The sum on the right-hand side is the representation mass of $K$ by $L$ defined in [Xu01]. The results in [Kne61], [Wei62] and [Sch84] (see also [Xu01]) imply that

$$
\mathfrak{Y} \text { is } \begin{cases}\text { Hardy-Littlewood } & \text { if } \operatorname{dim}(W) \geqslant 2, \\ \text { strongly Hardy-Littlewood } & \text { if } \operatorname{dim}(W) \geqslant 3 .\end{cases}
$$

When $m=1$, the above was pointed out in [BR95] and the idea used there is also similar to those in [Kne61], [Wei62] and [Sch84], where the action of the spin group of $V$ is utilized. In particular, in view of [Sch84] and [Xu01], when $\operatorname{dim}(W)=2$ the density function defined by the Kottwitz invariant in [BR95] should be interpreted by the spinor norm map. We explain this in the following.

First of all, we can extend the spinor norm map to $\mathfrak{Y}$. Indeed, fix a $x_{0} \in \mathfrak{Y}(\mathbb{Q})$ and consider an arbitrary point $x \in \mathfrak{Y}(\mathbb{Q})$. Since $S O(V)$ acts on $\mathfrak{Y}(\mathbb{Q})$ transitively, there is a $\sigma \in S O(V)$ such that $x=\sigma x_{0}$. We can define $\theta(x)=\theta(\sigma)$ in $\mathbb{Q}^{\times} / \theta(S O(W))$. It is well defined because the stabilizer of $x_{0}$ is conjugate to $S O(W)$. It can easily be checked that this map $\theta$ does not depend on the choice of $x_{0}$. Similarly, we can also define $\theta_{\mathrm{A}}: \mathfrak{Y}\left(\mathbf{A}_{\mathbb{Q}}\right) \longrightarrow \mathbf{I}_{\mathbb{Q}} / \theta_{\mathrm{A}}\left(S O_{\mathrm{A}}(W)\right)$. There is a quadratic character defined as

$$
\chi: \mathbf{I}_{\mathbb{Q}} / \mathbb{Q}^{\times} \theta_{\mathrm{A}}\left(S O_{\mathrm{A}}(W)\right) \longrightarrow\{ \pm 1\}, \quad \chi\left(\left(a_{p}\right)\right)=\prod_{p}\left(a_{p},-\operatorname{det}(W)\right),
$$

where $p$ runs over all primes including infinity. Then the density function $\delta(x)$ on $\mathfrak{Y}\left(\mathbf{A}_{\mathbb{Q}}\right)$ is simply $1+\chi \theta_{\mathrm{A}}$ by $[\mathrm{Xu01}$, Remarks 2.2 .3 and 2.2.4].

When $\operatorname{dim}(W) \leqslant 1, \mathfrak{Y}$ is still Hardy-Littlewood by [Xu01] (see [Xu01, Remark 2.2.3]). By the same argument as above, one has the following map induced by the adèlic spinor norm map: $\theta_{\mathrm{A}}$ : $\mathfrak{Y}\left(\mathbf{A}_{\mathbb{Q}}\right) \longrightarrow \mathbf{I}_{\mathbb{Q}} / \mathbf{I}_{\mathbb{Q}}^{2}$. Then the density function $\delta(x)$ is given by $\delta(x)=\frac{1}{2} \sum_{\chi} \chi \theta_{\mathrm{A}}$, where $\chi$ runs over all the characters of $\mathbf{I}_{\mathbb{Q}} / \mathbb{Q}^{\times} \theta_{\mathrm{A}}\left(S O_{\mathrm{A}}(L)\right)$.

When $\mathfrak{Y}$ is not strongly Hardy-Littlewood, it is natural to study the following constant which measures the difference between $N(T, \mathfrak{Y})$ and its Hardy-Littlewood expectation:

$$
c_{\mathfrak{Y}}=\lim _{T \rightarrow \infty} \frac{N(T, \mathfrak{Y})}{\mu_{\infty}(T, \mathfrak{Y})}\left(\prod_{p<\infty} \bar{\lambda}_{p}\left(\mathfrak{Y}\left(\mathbb{Z}_{p}\right)\right)^{-1}=\varepsilon \frac{r(K, \operatorname{cls}(L))}{r(K, \operatorname{gen}(L))},\right.
$$

and

$$
\varepsilon= \begin{cases}1 & \text { if } \operatorname{dim}(W)=2 \\ \frac{1}{2} & \text { if } \operatorname{dim}(W) \leqslant 1\end{cases}
$$

It is clear that

$$
0 \leqslant c_{\mathfrak{Y}} \leqslant \begin{cases}2 & \text { if } \operatorname{dim}(W)=2 \\ \frac{h}{2} & \text { if } \operatorname{dim}(W) \leqslant 1\end{cases}
$$

where $h$ is the class number of gen $(L)$.

For $\operatorname{dim}(W) \leqslant 1, c_{\mathfrak{Y}}=h / 2$ if and only if $\operatorname{cls}(L)$ is the only class in gen $(L)$ that represents $K$, and $c_{\mathfrak{Y}}=\frac{1}{2}$ if and only if every class in gen $(L)$ has the same representation mass for $K$.

For $\operatorname{dim}(W)=2$, we have

$$
c_{\mathfrak{Y}}= \begin{cases}2 & \text { if } K \text { is an exceptional lattice for } \operatorname{gen}(L), \\ 1 & \text { if } K \text { is not a splitting lattice for } \operatorname{gen}(L), \\ \text { neither } 1 \text { nor } 2 & \text { if } K \text { is splitting but not exceptional for } \operatorname{gen}(L),\end{cases}
$$




\section{REPRESENTATIONS OF SPINOR GENERA}

when $K$ is represented by $L$. We refer the readers to [Xu01, Definition 1.1.8] for the definitions of exceptional and splitting lattices. Exceptional lattices of any rank can be characterized by the relative spinor norm groups [HSX98]. Ternary splitting lattices are completely determined by [Xu01, Theorem 1.5.1]. It is easy to see that [Bor01, Theorem 0.2] follows from [Xu01, Theorem 1.5.1] as condition (2) in [Xu01, Theorem 1.5.1] is violated at infinity. Finally, we answer Question 0.3 in [Bor01] more explicitly by the following example.

Example 5.1. Let $f(x, y, z)=-9 x^{2}+2 x y+7 y^{2}+2 z^{2}$ and let $q$ be an odd prime which is inert in $\mathbb{Q}(\sqrt{2}) / \mathbb{Q}$. Since gen $(f)$ represents 1 by Example 3.8, $q^{2 m}$ is also represented by gen $(f)$. Then $q^{2 m}$ is a splitting number but not an exceptional number for gen $(f)$ by [Xu01, Theorem 1.5.1], [HSX98] and $[\mathrm{HSX}]$, or $[\mathrm{Sch} 80]$. Let $\mathfrak{Y}_{m}$ be the affine variety defined by the equation $f(x, y, z)=-9 x^{2}+$ $2 x y+7 y^{2}+2 z^{2}=q^{2 m}$. By $[\mathrm{Xu01}$, Remark 2.2.3], we have

$$
c_{\mathfrak{Y}_{m}}=1+\frac{\alpha_{q}^{+}\left(q^{2 m}, f\right)-\alpha_{q}^{-}\left(q^{2 m}, f\right)}{\alpha_{q}^{+}\left(q^{2 m}, f\right)+\alpha_{q}^{-}\left(q^{2 m}, f\right)}
$$

because $\alpha_{p}^{-}\left(q^{2 m}, f\right)=0$ at every $p \neq q$ by [HSX98, Theorem 5.1] and [Xu00, Theorem 2.1, Case II]. It is computed in [Sch84, Hilfssatz 8] that $\alpha_{q}^{+}\left(q^{2 m}, f\right)-\alpha_{q}^{-}\left(q^{2 m}, f\right)=(-1)^{m} q^{-1}(q-1)$. Since $\alpha_{q}^{+}\left(q^{2 m}, f\right)+\alpha_{q}^{-}\left(q^{2 m}, f\right)$ is the usual local density which is computed in [Sie35, Hilfssatz 16], we obtain

Therefore

$$
\alpha_{q}^{+}\left(q^{2 m}, f\right)+\alpha_{q}^{-}\left(q^{2 m}, f\right)=\left(1-q^{-2}\right)\left(\frac{1-\left(q^{-1}\right)^{m}}{1-q^{-1}}+\frac{\left(q^{-1}\right)^{m}}{1+q^{-1}}\right)
$$

$$
c_{\mathfrak{Y}_{m}}=1+(-1)^{m} \frac{q-1}{q+1-2 q^{-m}} .
$$

\section{ACKNOWLEDGEMENTS}

Both authors thank John Hsia and Rainer Schulze-Pillot for stimulating discussions. The research of the first author was partially supported by the National Science Foundation and the National Security Agency. The second author is supported by the Hundred Talent Program of the Chinese Academy of Sciences and Morningside Center of Mathematics.

\section{REFERENCES}

Bor01 M. Borovoi, On representations of integers by indefinite quadratic forms, J. Number Theory 90 (2001), 281-293.

BH82 J. W. Benham and J. S. Hsia, On spinor exceptional representations, Nagoya Math. J. 87 (1982), $247-260$.

BR95 M. Borovoi and Z. Rudnick, Hardy-Littlewood varieties and semi-simple groups, Invent. Math. 111 (1995), 37-66.

CF99 T. Chinburg and E. Friedman, An embedding theorem for quaternion algebras, J. London Math. Soc. (2) 60 (1999), 33-44.

DRS93 W. Duke, Z. Rudnick and P. Sarnak, Density of integer points on affine homogeneous varieties, Duke Math. J. 71 (1993), 143-179.

EM93 A. Eskin and C. McMullen, Mixing, counting, and equidistribution in Lie groups, Duke Math. J. 71 (1993), 181-209.

EMS96 A. Eskin, S. Mozes and N. Shah, Unipotent flows and counting lattice points on homogeneous varieties, Ann. Math. 143 (1996), 253-299.

EH82 D. R. Estes and J. S. Hsia, Exceptional integers of some ternary quadratic forms, Adv. Math. 45 (1982), 310-318. 


\section{REPRESENTATIONS OF SPINOR GENERA}

Hsi99 J. S. Hsia, Arithmetic of indefinite quadratic forms, Contemp. Math. 249 (1999), 1-15.

HSX98 J. S. Hsia, Y. Y. Shao and F. Xu, Representations of indefinite quadratic forms, J. Reine Angew. Math. 494 (1998), 129-140.

HSX J. S. Hsia, Y. Y. Shao and F. Xu, Spinor norms of relative local integral rotations, Preprint.

Kne61 M. Kneser, Darstellungsmasse indefiniter quadratischer Formen, Math. Z. 77 (1961), 188-194.

Ome58 O. T. O'Meara, The integral representations of quadratic forms over local fields, Am. J. Math. 80 (1958), 843-878.

Ome73 O. T. O'Meara, Introduction to quadratic forms (Springer, Berlin, 1973).

Sie35 C. L. Siegel, Über die analytische Theorie der quadratischen Formen. I, Ann. Math. 36 (1935), $527-606$.

Sch80 R. Schulze-Pillot, Darstellung durch Spinorgeschlechter ternärer quadratischer Formen, J. Number Theory 12 (1980), 529-540.

Sch84 R. Schulze-Pillot, Darstellungsmasse von Spinorgeschlechtern ternärer quadratischer Formen, J. Reine Angew. Math. 352 (1984), 115-132.

Wei62 A. Weil, Sur la théorie des formes quadratiques, in Colloque sur la théorie des groupes algébriques, Brussels, 1962 (Librairie Universitaire, Louvain, and Gauthier-Villars, Paris, 1962), 9-22.

Xu99 F. Xu, Arithmetic Springer theorem on quadratic forms under field extensions of odd degree, Contemp. Math. 249 (1999), 175-197.

Xu00 F. Xu, Representations of indefinite ternary quadratic forms over number fields, Math. Z. 234 (2000), $115-144$.

Xu01 F. Xu, Representation masses of spinor genera, Duke Math. J. 110 (2001), 279-308.

Wai Kiu Chan wkchan@wesleyan.edu

Department of Mathematics, Wesleyan University, Middletown, CT 06459, USA.

Fei Xu xufei@math.ac.cn

Academy of Mathematics and System Sciences, Chinese Academy of Sciences, Beijing 100080, People's Republic of China. 